\title{
Trans-upper-sternal Approach to the Cervicothoracic Junction
}

\author{
Yi-Lin Liu MD, Ying-Jie Hao MD, Tao Li MD, \\ Yue-Ming Song MD, Li-Min Wang MD
}

Received: 8 October 2007 / Accepted: 6 August 2008/Published online: 28 August 2008

(C) The Association of Bone and Joint Surgeons 2008

\begin{abstract}
From August 1999 to February 2006, 11 patients with cervicothoracic lesions (eight males, three females; age range, 17-77 years) were surgically treated using the trans-upper-sternal approach. Combined cervicothoracic incision and upper sternotomy facilitated exposure for tumor resection, partial or subtotal removal of the involved vertebrae, and spinal cord decompression. The spinal column then was stabilized. Neurologic status was assessed using the Frankel classification. Followup for a minimum of 10 months (mean, 31 months; range, 1056 months) revealed one patient had a chyle leak $(50 \mathrm{~mL})$ 1 day after surgery, which resolved after 2 days of drainage. One patient had a transient vocal cord paresis, which recovered within 3 months of surgery. All the patients had improved neurologic function. No nonunions or instrument-related complications developed. Stability of the vertebral column was maintained during followup in all patients. The trans-upper-sternal approach can provide
\end{abstract}

Each author certifies that he or she has no commercial associations (eg, consultancies, stock ownership, equity interest, patent/licensing arrangements, etc) that might pose a conflict of interest in connection with the submitted article.

Each author certifies that his or her institution has approved the reporting of these cases, that all investigations were conducted in conformity with ethical principles of research, and that informed consent for participating in the study was obtained.

Y.-L. Liu, T. Li, Y.-M. Song ( $\varangle)$

Department of Orthopedic Surgery, West China Hospital,

Sichuan University, No. 37 GuoXue Road, Chengdu,

Sichuan 610041, China

e-mail: liu200888@yahoo.com.cn

Y.-J. Hao, L.-M. Wang

Department of Orthopedic Surgery, the First Affiliated Hospital,

Zhengzhou University, Zhengzhou, China excellent exposure for reconstruction of the cervicothoracic junction. Special care must be taken to avoid injury to the recurrent laryngeal nerve and the thoracic duct.

Level of Evidence: Level IV, therapeutic study. See the Guidelines for Authors for a complete description of levels of evidence.

\section{Introduction}

The cervicothoracic junction [12] (CTJ) refers to the region between the sixth cervical and the third thoracic vertebrae. In those vertebral segments, physiologic cervical lordosis and thoracic kyphosis occur creating a unique biomechanical feature. Most cervicothoracic lesions involve the anterior aspect of the spinal cord and cause vertebral body destruction, spinal kyphosis, and ventral spinal cord compression. The anterior approach often is used for treatment of lesions in this region $[4,8,15,18]$. The anterior aspect of the CTJ is a difficult region to approach in spinal surgery because in this region, there are many vital structures that can hinder the surgical approach [11]. The exposure is made more difficult by vertebral body destruction with kyphotic deformity and structural instability $[6,11,19]$.

Several approaches to the CTJ have been described [3, $8,10,12,15,18,21]$. Each approach has its advocates, but all the approaches have some limitations. The anterior low cervical approach to the CTJ is familiar to most spinal surgeons. By this approach, C7 to T1 vertebrae can be exposed adequately, but an exposure distal to this is limited by the sternum, clavicle, and kyphotic alignment of the upper thoracic spine [18]. Also, there may be a potential increase in the approach-related injury to the recurrent laryngeal nerve, vascular structures, and esophagus. This approach does not permit optimal placement of the caudal 
end of the structural graft and adequate caudal screw fixation of the anchoring plate. Trap-door exposure [18] allows gross total resection of tumors with possible exposure from $\mathrm{C} 3$ to $\mathrm{T} 4$. This approach, which combines the supraclavicular approach with sternotomy and the anterolateral approach (anterior thoracotomy), achieves anterior observation and reduces the risk related to the spinal cord manipulation. However, this approach still carries risks associated with thoracotomy $[10,14]$. A direct sternumsplitting approach affords extensile exposure and control over the great vessels but has high operative mortality and morbidity $[8,12,21]$. Also, the lower half of the sternotomy exposure is not required [14]. Sundaresan et al. [21] repopularized the transsternal approach with resection of the manubrium sterni and one-third medial portion of the clavicle. The resection of the clavicle considerably improves exposure and provides a strut graft. Others argue, however, that it is not necessary to perform the transverse incision of the approach to the contralateral neck [12]. The combined anterolateral cervical and posterior transpleural transthoracic approach is for exposure from C3 to T9 [15]. Although the exposure can provide a larger operative field, internal fixation may be difficult to perform. Also, additional danger during entrance to the chest is present with this technique [12].

Based on anatomic studies that we performed on 40 adult cadavers, we modified the full sternotomy [3] with a trans-upper-sternal approach for treatment of cervicothoracic lesions. We propose that this modified technique can be a better approach to gain access to the CTJ. We also propose that this improved exposure will result in better clinical outcomes of surgery directed at CTJ lesions and injury.

\section{Materials and Methods}

From August 1999 to February 2006, 11 selected patients with cervicothoracic lesions underwent surgery using the trans-upper-sternal approach. The medical records of the patients were reviewed retrospectively, and patients' age, gender, diagnosis, surgical procedure, followup, neurologic examinations before and after surgery, and final outcome were recorded (Table 1). The inclusion criteria were: (1) the lesion should involve the CTJ; (2) the caudal normal vertebra selected for instrumentation should be below the

Table 1. Patient data

\begin{tabular}{|c|c|c|c|c|c|c|}
\hline $\begin{array}{l}\text { Patient } \\
\text { number }\end{array}$ & $\begin{array}{l}\text { Age (years)/ } \\
\text { Gender }\end{array}$ & Diagnosis & Procedure & $\begin{array}{l}\text { Followup } \\
\text { (months) }\end{array}$ & $\begin{array}{l}\text { Frankel grade } \\
\text { (initial/followup) }\end{array}$ & Outcome \\
\hline 1 & $52 / \mathrm{M}$ & Unknown primary metastasis to $\mathrm{T} 3$ & $\begin{array}{l}\mathrm{T} 3 \text { corpectomy, autograft }+ \text { plate- } \\
\text { screw at } \mathrm{T} 2-\mathrm{T} 4\end{array}$ & 12 & $\mathrm{~B} / \mathrm{D}$ & Good \\
\hline 2 & $35 / F$ & Fibrolipoangioma at C6-T1 & Resection of C6-T1 fibrolipoangioma & 39 & $\mathrm{D} / \mathrm{E}$ & Excellent \\
\hline 3 & $67 / \mathrm{M}$ & Plasmacytoma at $\mathrm{T} 1$ & $\mathrm{~T} 1$ corpectomy, autograft at C7-T2 & 56 & $\mathrm{C} / \mathrm{D}$ & Fair \\
\hline 4 & $41 / \mathrm{M}$ & Tuberculosis at $\mathrm{T} 1-\mathrm{T} 2$ & $\begin{array}{l}\text { T1-T2 corpectomy, } \\
\text { autograft }+ \text { titanium cylinder } \\
\text { with plate-screw at T2-T4 }\end{array}$ & 30 & $\mathrm{C} / \mathrm{D}$ & Good \\
\hline 5 & $17 / \mathrm{M}$ & Aneurysmal bone cyst at $\mathrm{C} 7$ & $\begin{array}{l}\text { C7 corpectomy, autograft with } \\
\text { plate-screw at C6-T1 }\end{array}$ & 16 & $\mathrm{C} / \mathrm{E}$ & Excellent \\
\hline 6 & $52 / \mathrm{M}$ & Esophageal metastasis to C6-T1 & $\begin{array}{l}\text { C6-T1 partial corpectomy, } \mathrm{C} 7 \\
\text { corpectomy, allograft }+ \text { titanium } \\
\text { cylinder with plate-screw at } \mathrm{C} 7-\mathrm{T} 2\end{array}$ & 38 & $\mathrm{C} / \mathrm{E}$ & Good \\
\hline 7 & $61 / \mathrm{F}$ & Lung metastasis to $\mathrm{T} 1$ & $\begin{array}{l}\mathrm{T} 1 \text { corpectomy, allograft }+ \text { titanium } \\
\text { cylinder with plate-screw at C7-T2 }\end{array}$ & 15 & $\mathrm{~B} / \mathrm{C}$ & Good \\
\hline 8 & $25 / \mathrm{F}$ & $\mathrm{T} 1$ fracture & $\begin{array}{l}\mathrm{T} 1 \text { corpectomy, autograft with } \\
\text { plate-screw at } \mathrm{C} 7-\mathrm{T} 2\end{array}$ & 10 & $\mathrm{D} / \mathrm{E}$ & Excellent \\
\hline 9 & $35 / \mathrm{M}$ & C7-T1 dislocation & $\begin{array}{l}\text { C7-T1 discectomy, autograft with } \\
\text { plate-screw at C7-T1 }\end{array}$ & 35 & $\mathrm{~B} / \mathrm{D}$ & Good \\
\hline 10 & $33 / \mathrm{M}$ & C7 fracture, C6-C7 dislocation & $\begin{array}{l}\text { C7 corpectomy, autograft with } \\
\text { plate-screw at C6-T1 }\end{array}$ & 37 & $\mathrm{D} / \mathrm{E}$ & Excellent \\
\hline 11 & $38 / \mathrm{M}$ & $\mathrm{T} 1$ fracture, herniated $\mathrm{C} 5-\mathrm{C} 6$ disc & $\begin{array}{l}\text { C5-C6 discectomy, T1 partial } \\
\text { corpectomy, autograft with } \\
\text { plate-screw at C7-T1 }\end{array}$ & 49 & $\mathrm{~B} / \mathrm{D}$ & Good \\
\hline
\end{tabular}

$\mathrm{M}=$ male $\mathrm{F}=$ female. 
level of the clavicles on the lateral radiograph; and (3) surgical fusion should be achieved. Patients were excluded if the lateral radiograph showed that the normal, caudal vertebra selected for instrumentation was above the level of the clavicles. There were eight males and three females aged 17 to 67 years (average, 42 years). Their disease courses ranged from 10 days to 24 months. Their spinal lesions were located in: $\mathrm{T} 3$ (one patient), $\mathrm{C} 7$ to $\mathrm{T} 1$ (one patient), T1 to T2 (one patient), C7 (two patients), C6 to T1 (two patients), and T1 (four patients). Four patients had traumatic lesions (fractures in two, dislocation in one, and fracture-dislocation in one); six patients had tumors (metastatic neoplasms in three [lung carcinoma, esophageal carcinoma, primary lesions of unknown origin], and primary neoplasms in three [plasmacytoma, fibrolipoangioma, aneurysmal bone cyst]); and one patient had tuberculosis. Plain radiographs, computed tomographic scans (CT), and MR images were obtained in all patients.

Based on the classification of Frankel et al. [7], the patients' neurologic status was assessed on admission and at final followup. All the patients had neurologic deficits on admission (Table 1). Among them, three had an incomplete cord syndrome (anterior cord syndrome) and eight had root deficits. On admission, four patients' neurologic status was classified as Frankel B, four as Frankel C, and three as Frankel D.

General endotracheal anesthesia was used in all cases. Patients were positioned supine with a roll placed between the scapulae to facilitate retraction of the shoulders. The neck was extended and slightly turned to the right. The shoulders were pulled caudally using a broad tape to allow a less hindered view of the spine during intraoperative radiography. The incision was made along the medial border of the inferior half of the left sternocleidomastoid muscle and extended down to the suprasternal notch. The lower part of the incision then extended in the anterior midline to the lower border of the sternal angle ending at the level between the second and the third ribs (Fig. 1).

After the skin was incised, the dissection was continued through the platysma, creating subcutaneous flaps. The dissection proceeded along the medial border of the sternocleidomastoid muscle. The interclavicular ligament, sternohyoid and sternothyroid muscles were cut. After subperiosteal exposure of the sternum, blunt digital dissection was used to mobilize the retrosternal soft tissues and the rest of the thymus. The sternum was split in the midline with the sternotomy saw to a point $2 \mathrm{~cm}$ distal to the sternal angle. The two parts of the split sternum then were cut transversely (Fig. 2) between the second and third ribs. Bleeding from the sternum was stopped by bone wax and a small, self-retaining retractor was positioned between the vertical cuts of the manubrium to create a larger retrosternal space. The esophagus and the trachea then were

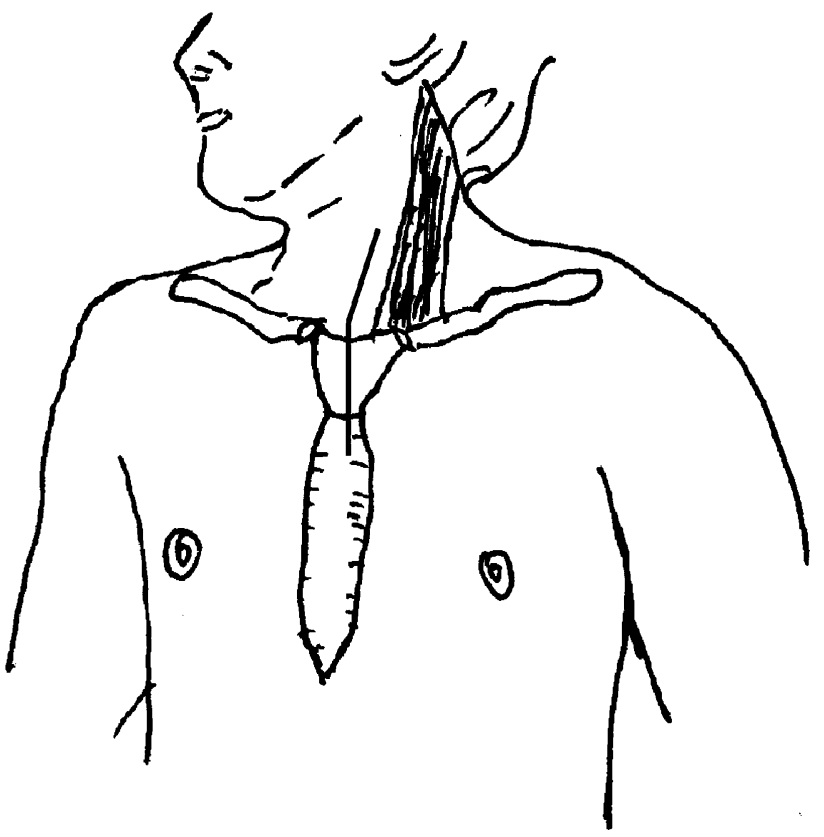

Fig. 1 The incision was made along the inferomedial border of the left sternocleidomastoid muscle down to the suprasternal notch and then extended in the midsagittal plane onto the lower border of the sternal angle ending at the level between the second and third ribs.

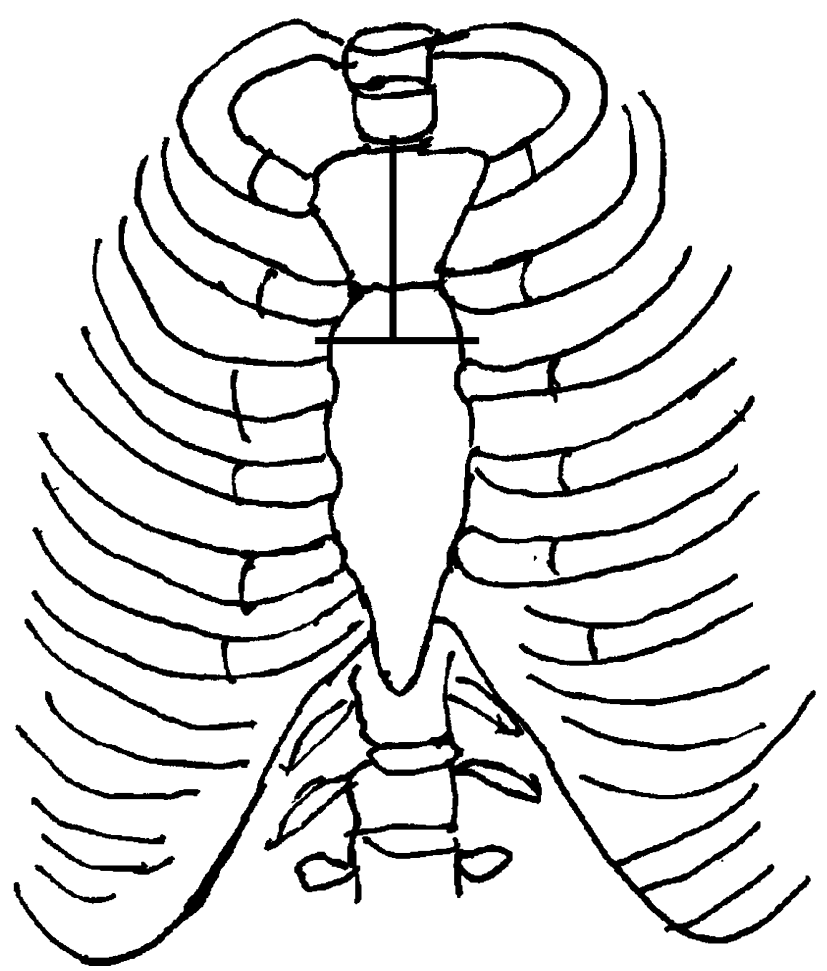

Fig. 2 A median sternotomy was performed distally just $2 \mathrm{~cm}$ below the sternal angulus, and the two parts of the split sternum were completely cut transversely.

retracted together with the recurrent laryngeal nerve, and the left common carotid sheath and the left jugular vein were gently retracted laterally. 
As the prevertebral fascia was dissected free, the vertebral bodies from C6 to T3 were observed. Identification of the longus colli muscles, which insert consistently into the lateral aspects of the T1, T2, and T3 vertebral bodies, helps to orient the surgeon to the level of dissection. A lateral radiograph always should be taken with a needle inserted into a proximal disc space to ensure identification of the proper level. Care was taken to protect the left recurrent laryngeal nerve and the thoracic duct. In this series, surgical procedures including tumor resection, corpectomy, spinal decompression, vertebral reconstruction, and anterior platescrew fixation were performed. After the orthopaedic procedure, a tube was placed behind the sternum for routine drainage and the manubriotomy was closed using a strong nonabsorbable suture material for the vertical and transverse limbs. The sternohyoid and sternothyroid muscles were resutured at their distal attachment. The wound was closed in a layered fashion. After surgery, the patients wore a cervicothoracic brace for 3 months.

The minimum postoperative followup was 10 months (mean, 31 months; range, 10-56 months). Followups included a physical examination, plain radiography, and CT scans. All radiographs were viewed by two individuals (HD, JZ) who were blinded to the results. We identified bony fusion by the presence of bridging bone across the diseased vertebrae [9]. Surgery-related complications and symptoms also were noted.

The patient outcomes were assessed according to a scale described by Sapkas et al. [20], and the outcomes were classified as excellent if there was complete recovery and return to previous activity level; good if there was occasional pain but return to gainful activity and only intermittent use of analgesics; fair for partial recovery, frequent use of analgesics, and activity affected; or poor if there was no relief of the original symptoms, constant pain, and full-time support required.

\section{Results}

Blood loss ranged from 200 to $1000 \mathrm{~mL}$ (average, $550 \mathrm{~mL}$ ), and the duration of surgery ranged from 2 to 5 hours. There were no pleural injuries, vascular lesions, or cardiac complications. One patient had a chyle leak $(50 \mathrm{~mL}) 1$ day after surgery, which disappeared after 2 days of drainage (Patient 1, Table 1). One patient had transient vocal cord paresis subsequent to injury to the recurrent laryngeal nerve, which resolved within 3 months after surgery (Patient 4, Table 1). One patient with a primary neoplasm, of unknown origin, in $\mathrm{T} 3$, died from general metastasis and multiorgan system failure 12 months after surgery. Despite this, the patient remained ambulatory until his death (Patient 1, Table 1) (Fig. 3).
One patient with a $\mathrm{T} 1$ plasmocytoma died 4 years after surgery owing to tumor recurrence and pulmonary infection (Patient 3, Table 1).

The patients with incomplete cord injury had considerably improved neurologic function (Table 1). All patients with root deficits also were improved, and 6 months after surgery all recovered completely. Using the assessment scale described by Sapkas et al. [20], four patients had excellent results, six had good results, and one had a fair result. Bony fusion was achieved within 3 to 4 months postoperatively. Nonunion and instrument-related complications were not observed.

\section{Discussion}

Addressing spinal lesions around the CTJ is technically challenging because the cervicothoracic vertebral bodies are relatively inaccessible owing to the anatomic structures that impede the anterior approach [21]. Various approaches have been suggested to provide safe and effective access to this region $[3,8,10,12,13,15,18]$. Our small series suggests the trans-upper-sternal approach is a technically feasible, less invasive, and effective exposure to gain access to the CTJ. This approach provided an excellent clinical outcome with less surgical morbidity.

Our study has some limitations. The main limitations were small sample size and retrospective study design. The small sample was the result of strict inclusion criteria. The lack of a control group did not allow for comparison of results. Also, we did not include radiographic analysis; therefore, the potential for bone nonunion and instrumentrelated complications could not be assessed.

Compared with full or other partial sternotomy [3, 18, 21], the trans-upper-sternal approach to the CTJ is a relatively less invasive but more effective approach. Dissection can be performed between the tissue planes with no major structures resected, and control of the great vessels is easy to establish. The sternum is cut only partially and thoracotomy or osteotomy of the clavicle is not required thus avoiding complications of the lungs and pseudarthrosis [3, $12,18]$. This approach can provide a direct and good working field and avoid extensive dissection of vascular and neural structures in the cervicothoracic region. It allows corpectomy, discectomy, anterior decompression, maintenance of the anatomic alignment, fusion with the bone graft, and internal fixation with few complications. The recurrent laryngeal nerve has a longer route and has a relatively fixed anatomy in the tracheoesophageal groove, but the right side has substantial variation [5]. Therefore, left exposure by the trans-upper-sternal approach is preferable to decrease the risk of iatrogenic recurrent laryngeal nerve injury. 
Fig. 3A-D A 54-year-old man was admitted because of a 40day history of weakness in both lower extremities with evidence of metastasis to T3. He had paraparesis (1/5) and hypesthesia below the T5 dermatome. (A) The preoperative T2-weighted MR image shows a pathologic fracture and a ventral spinal cord compression in T3; and (B) preoperative CT shows gross bone destruction of the vertebral body of T3. After the T3 corpectomy, an iliac crest autogenous bone graft fusion with anterior platescrew fixation was performed using the trans-uppersternal approach. Histopathologic evaluation revealed adenocarcinoma of unknown primary. (C) Anteroposterior and (D) lateral plain radiographs obtained after surgery show the anterior platecrew instrumentation between $\mathrm{T} 2$ and $\mathrm{T} 4$.
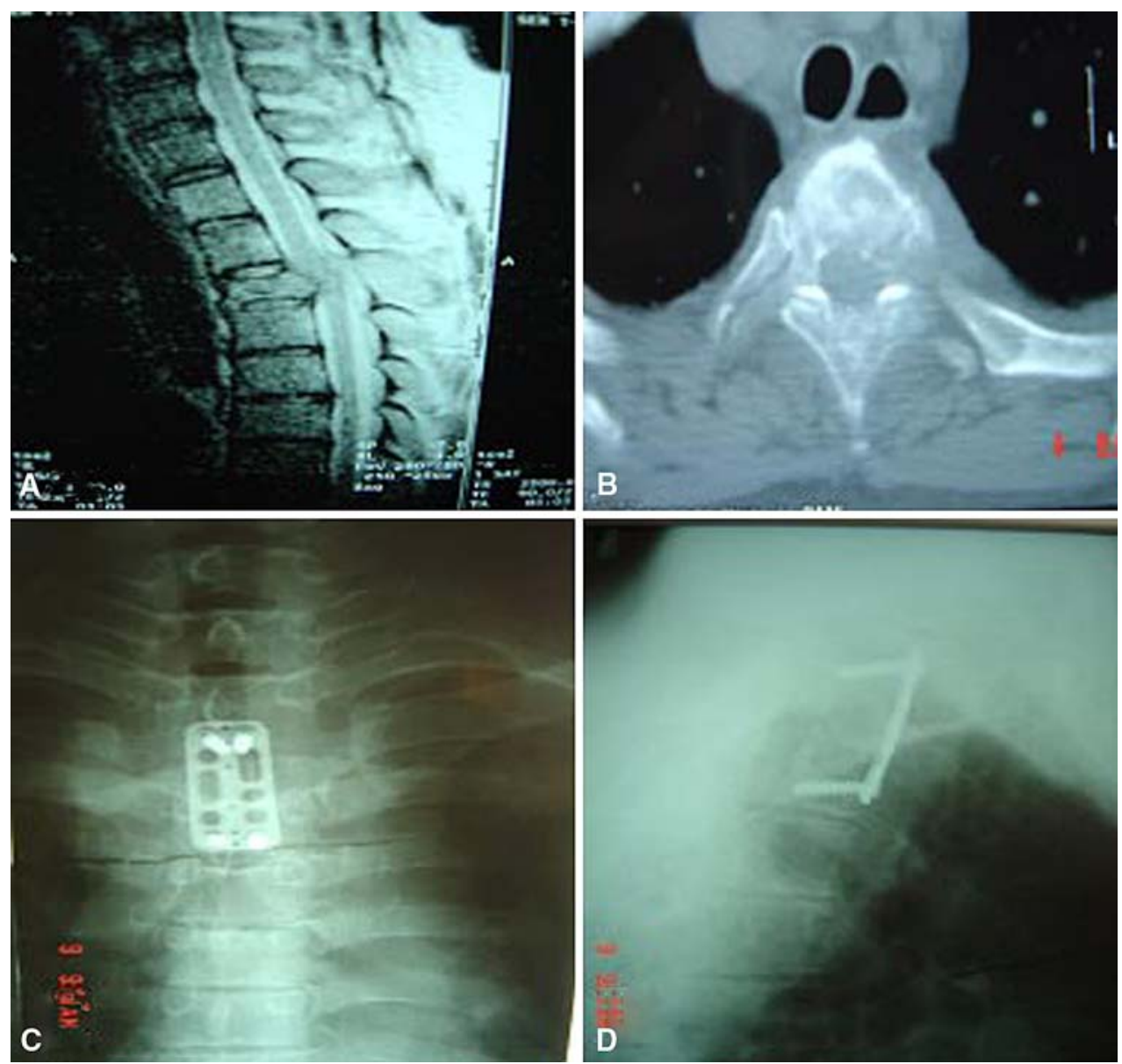

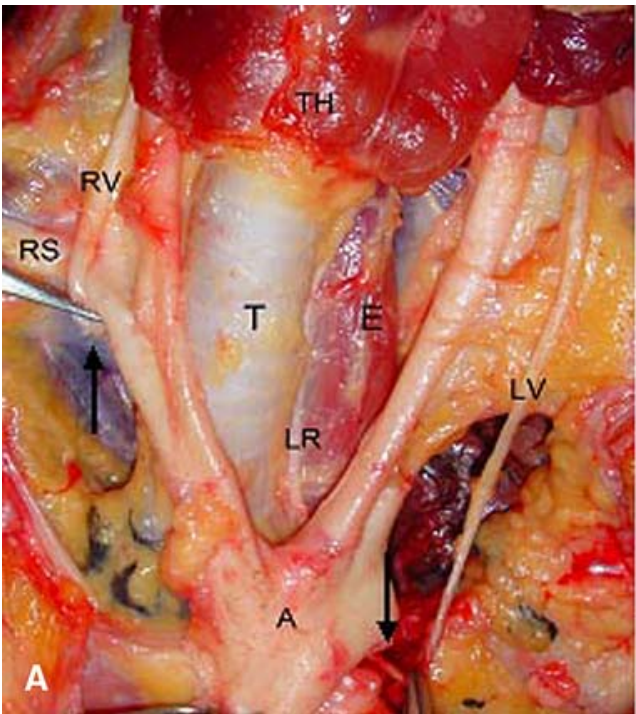

Fig. 4A-B (A) and (B) views show the microsurgical anatomy of the recurrent laryngeal nerve in the cervicothoracic junction. (A) The right recurrent laryngeal nerve loop is under the right subclavian artery, but the left side arises in front of the aortic arch. (B) Both of them return to the neck and ascend in the esophagotracheal groove.

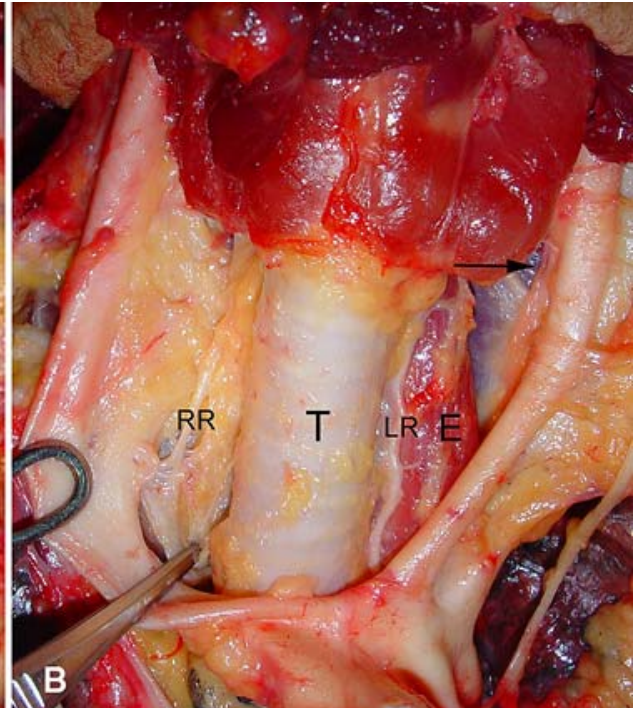

The legends show the: (LR) left recurrent laryngeal nerve, (RR) right recurrent laryngeal nerve, $(\mathrm{LV})$ left vagus nerve, $(\mathrm{RV})$ right vagus nerve, (A) aortic arch, (RS) right subclavian artery, (T) trachea, (E) esophagus, $(\mathrm{TH})$ thyroid, $(\rightarrow)$ inferior thyroid artery and vein, $(\uparrow \downarrow)$ recurrent point of the recurrent laryngeal nerve. 
The most frequent complication related to the anterior approach to the CTJ is vocal cord paresis secondary to injury of the recurrent laryngeal nerve [13] with the reported incidence ranging from $4.76 \%$ to $16.67 \%$ [16, 17]. Morbidity of the recurrent laryngeal nerve has been attributed to numerous factors, including direct insult during sharp dissection, overstretching of the nerves [5] especially at the recurrent point of the recurrent laryngeal nerve, ligation of the inferior thyroid vessels without identification of the nerve, anatomic variations of the nerve, compression of the recurrent laryngeal nerve by the endotracheal tube [1], or soft tissue swelling and hematoma [5]. One of our patients had transient vocal cord paresis after an injury to the recurrent laryngeal nerve, which resolved within 3 months after surgery (Patient 4, Table 1). Better understanding of the microsurgical anatomy and accurate recognition of possible areas of recurrent laryngeal nerve vulnerability can decrease iatrogenic injury to the recurrent laryngeal nerve (Fig. 4). Enough attention should be paid to avoiding retraction of the recurrent laryngeal nerve for an extended time or with a sharp apparatus (Fig. 5).

Left exposure of trans-upper-sternal approach poses the potential of injuring the thoracic duct. Ascending on the left side of the thoracic inlet and the esophagus, the thoracic duct passes behind the left subclavian. Forming an arch at $\mathrm{C} 7$ behind the carotid sheath anterior to the anterior scale muscle, it turns forward, flowing into the internal jugular vein. The thoracic duct most often is found between C7 and T1 within a triangle bounded medially by the longus colli muscles and the esophagus and posteriorly by the first rib. It is not necessary to dissect the thoracic duct with the trans-upper-sternal approach to the CTJ; it can be retracted laterally and protected with the left common carotid and internal jugular vein. If intraoperative damage

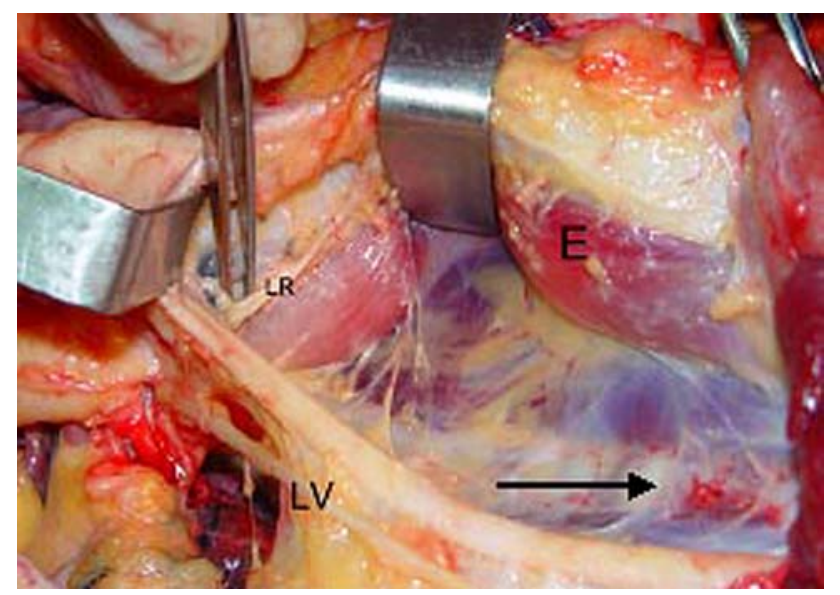

Fig. 5 Retraction for an extended time or with a sharp apparatus of the recurrent laryngeal nerve (LR) and the esophagus (E) should be avoided. The arrow denotes an exposure of the cervicothoracic region. is suspected, chyle will appear a short time later at the leakage in the operation situs and the thoracic duct should be doubly ligated proximally and distally [2]. Because of intraoperative injury to the lateral branch of the thoracic duct, a chyle leak $(50 \mathrm{~mL})$ developed in one of our patients 1 day after surgery. The leak disappeared after 2 days of drainage (Patient 1, Table 1).

The trans-upper-sternal approach can provide an excellent exposure of the CTJ. This approach has advantages of being technically feasible, less invasive, and effective for anterior decompression, maintenance of anatomic alignment, fusion with the bone graft, and internal fixation with fewer complications and traumatic lesions. Care must be taken to avoid injury to the recurrent laryngeal nerve and thoracic duct.

Acknowledgments We thank Drs. Hong Duan and Jiancheng Zeng, Department of Orthopaedics, West China Hospital, Chengdu, Sichuan province, China, for assistance with radiograph, CT, and MR imaging data.

\section{References}

1. Apfelbaum RI, Kriskovich MD, Haller JR. On the incidence, cause, and prevention of recurrent laryngeal nerve palsies during anterior cervical spine surgery. Spine. 2000;25:2906-2912.

2. Boockvar JA, Philips MF, Telfeian AE, O'Rourke DM, Marcotte PJ. Results and risk factors for anterior cervicothoracic junction surgery. J Neurosug. 2001;94(1 suppl):12-17.

3. Cauchoix J, Binet J. Anterior surgical approaches to the spine. Ann R Coll Surg Engl. 1957;21:234-243.

4. Darling GE, McBroom R, Perrin R. Modified anterior approach to the cervicothoracic junction. Spine. 1995;20:1519-1521.

5. Ebraheim NA, Lu J, Yang H, Heck BE, Yeasting RA. Vulnerability of the sympathetic trunks during the anterior approach to the lower cervical spine. Spine. 2000;25:1603-1606.

6. Evans DK. Dislocations at the cervicothoracic junction. $J$ Bone Joint Surg Br. 1983;65:124-127.

7. Frankel HL, Hancock DO, Hyslop G. The value of postural reduction in the initial management of closed injuries of the spine with paraplegia and tetraplegia. Paraplegia. 1969;7:179-192.

8. Hodgson AR, Stock FE, Fang HS, Ong GB. Anterior spinal fusion: the operative approach and pathological findings in 412 patients with Pott's disease of the spine. Br J Surg. 1960;48:172178.

9. Jayaswal A, Upendra B, Ahmed A, Chowdhury B, Kumar A. Video-assisted thoracoscopic anterior surgery for tuberculous spondylitis. Clin Orthop Relat Res. 2007;460:100-107.

10. Kaya RA, Turkmenoglu ON, Koc ON, Gene HA, Cavusoglu H, Ziyal IM, Aydin Y. A perspective for the selection of surgical approaches in patients with upper thoracic and cervicothoracic junction instabilities. Surg Neurol. 2006;65:454-463; discussion 463.

11. Knoller SM, Brethner L. Surgical treatment of the spine at the cervicothoracic junction: an illustrated review of a modified sternotomy approach with the description of tricks and pitfalls. Arch Orthop Trauma Surg. 2002;122:365-368.

12. Kurz LT, Pursel SE, Herkowitz HH. Modified anterior approach to the cervicothoracic junction. Spine. 1991;16(10 suppl):S542547. 
13. Le Huec JC, Lesprit E, Guibaud JP, Gangnet N, Aunoble S. Minimally invasive endoscopic approach to the cervicothoracic junction for vertebral metastases: report of two cases. Eur Spine 2001;10:421-426.

14. Luk KD, Cheung KM, Leong JC. Anterior approach to the cervicothoracic junction by unilateral or bilateral manubriotomy: a report of five cases. J Bone Joint Surg Am. 2002;84: 1013-1017.

15. Micheli LJ, Hood RW. Anterior exposure of the cervicothoracic spine using a combined cervical and thoracic approach. J Bone Joint Surg Am. 1983;65:992-997.

16. Mihir B, Vinod L, Umesh M, Chaudhary K. Anterior instrumentation of the cervicothoracic vertebrae: approach based on clinical and radiologic criteria. Spine. 2006;31:E244-249.
17. Mulpuri K, LeBlanc JG, Reilly CW, Poskitt KJ, Choit RL, Sahajpal V, Tredwell SJ. Sternal split approach to the cervicothoracic junction in children. Spine. 2005;30:E305-310.

18. Nazzaro JM, Arbit E, Burt M. "Trap door" exposure of the cervicothoracic junction: technical note. J Neurosurg. 1994;80: 338-341.

19. Nichols CG, Young DH, Schiller WR. Evaluation of cervicothoracic junction injury. Ann Emerg Med. 1987;16:640-642.

20. Sapkas G, Papadakis S, Katonis P, Roidis N, Kontakis G. Operative treatment of unstable injuries of the cervicothoracic junction. Eur Spine J. 1999;8:279-283.

21. Sundaresan N, Shah J, Foley KM, Rosen G. An anterior surgical approach to the upper thoracic vertebrae. J Neurosurg. 1984;61: 686-690. 